\title{
The sequence of Compton dominance in blazars based on data from WISE and Fermi-LAT
}

\author{
Krzysztof Nalewajko and Maitrayee Gupta
}

\begin{abstract}
Nicolaus Copernicus Astronomical Center, Polish Academy of Sciences, Bartycka 18, 00-716 Warsaw, Poland e-mail: knalew@camk.edu.pl
\end{abstract}

Received 7 June 2017 / Accepted 6 August 2017

\begin{abstract}
The two-component broad-band spectral energy distributions of blazars were suggested to form a sequence in which (1) the peak frequency of the low-energy (synchrotron) component $v_{\mathrm{syn}}$ is anticorrelated with the bolometric synchrotron luminosity $L_{\mathrm{syn}}$ and (2) the luminosity ratio of the high-energy (inverse Compton) to synchrotron components $q=L_{\mathrm{IC}} / L_{\text {syn }}$ (Compton dominance) increases with $L_{\text {syn }}$ from the BL Lac objects (BL Lacs) to the flat spectrum radio quasars (FSRQs). The Compton dominance parameter is an important probe of plasma magnetisation in the blazar zones within relativistic jets. We investigate a sample of blazars detected by WISE in the mid-infrared (MIR) band and by Fermi-LAT in the GeV gamma-ray band, with the focus on the distribution of luminosities and photon indices. Our findings are the following: (1) the MIR photon index $\Gamma_{W 12}$ is a useful probe of the blazar sequence, with the exception of low-luminosity BL Lacs that are most likely contaminated by their host galaxies $\left(L_{W 1} \sim 10^{44} \mathrm{erg} \mathrm{s}^{-1}\right.$ and $\Gamma_{W 12}<1$ ); (2) $\Gamma_{W 12}$ is correlated with the gamma-ray photon index $\Gamma_{1-100 \mathrm{GeV}}$, with the MIR luminosity $L_{W 1}$, and with the Fermi/WISE Compton dominance $q_{\mathrm{FW}}=L_{1 \mathrm{GeV}} / L_{W 1}$; (3) a clean separation between FSRQs and BL Lacs can be seen in the parameter space of $\Gamma_{W 12}$ and $q_{\mathrm{FW}}$; and (4) the observed distribution of MIR luminosity $L_{W 1}$ versus Compton dominance $q_{\mathrm{FW}}$ for the entire sample of blazars can be modelled as a sequence of lepto-magnetic jet powers in the range $\log _{10} P_{\mathrm{eB}} \in[42: 45]$ with the preference for subequipartition magnetic fields $P_{\mathrm{B}} / P_{\mathrm{e}} \in[0.05: 1]$, assuming fixed bulk Lorentz factor $\Gamma_{\mathrm{j}}=15$, fixed jet opening angle $\Gamma_{\mathrm{j}} \Theta_{\mathrm{j}}=0.3$, fixed radiative efficiency of jet electrons $\epsilon_{\mathrm{em}}=50 \%$, and that external radiation luminosity scales like $L_{\mathrm{ext}} \propto P_{\mathrm{eB}}^{1.6}$ (parameter degeneracies are discussed).
\end{abstract}

Key words. quasars: general - BL Lacertae objects: general - galaxies: jets

\section{Introduction}

Blazars belong to the most luminous cosmic sources in terms of apparent luminosity. Their spectral energy distributions (SEDs) are dominated by non-thermal emission forming two main components: the synchrotron component extending between the radio and UV bands (in the case of BL Lac objects extending to the X-ray band), and the inverse Compton component (in the leptonic scenarios) dominating in the gamma-ray band (see Madejski \& Sikora 2016, for recent review). In the basic leptonic models of blazar emission, both of these components are produced by the same population of ultra-relativistic electrons accelerated in the so-called blazar zone in a relativistic jet. The physics of energy dissipation and particle acceleration in relativistic jets remains elusive. Two particular mechanisms are discussed most frequently: relativistic shock waves and relativistic magnetic reconnection. It is thought that their efficiency can be determined mainly from the composition of jets, and particularly from their magnetisation $\sigma=B^{2} /(4 \pi w)$, where $w$ is the relativistic specific enthalpy. While shock waves are more efficient at low magnetisations $(\sigma<1)$, magnetic reconnection is more efficient at high magnetisations $(\sigma>1)$. A very important argument was given by Sironi et al. (2015): both in the case of shocks and reconnection, the emitting regions are distinct from their background, and always closer to equipartition between the magnetic and kinetic energies $(\sigma \sim 1)$. Observations of blazars have been argued to be roughly consistent with equipartition (Böttcher et al. 2013; Dermer et al. 2014), however, leptonic models of BL Lac objects often imply matterdominated (by factor up to $~ 100$ ) jets (Tavecchio et al. 2011; Tavecchio \& Ghisellini 2016). Nevertheless, observations cannot reveal the magnetisation of the background jet plasma. This also implies that the blazar-emitting regions should not be characterised by high magnetisation values $(\sigma>1)$.

In luminous blazars, the dominant source of soft photons for Comptonization into the observed gamma-ray emission originates externally to the jet. Depending on the distance scale for the location of the emitting region along the jet, it could be either direct radiation from the accretion system (Dermer et al. 1992), broad emission lines (Sikora et al. 1994), or thermal emission of the hot dust (Błażejowski et al. 2000). In any case, the apparent gamma-ray luminosity is proportional to the energy density of the external radiation converted into the jet co-moving frame $L_{\gamma} \propto u_{\mathrm{ext}}^{\prime}$. On the other hand, the apparent luminosity of the synchrotron component is proportional to the local magnetic energy density $L_{\mathrm{syn}} \propto u_{\mathrm{B}}^{\prime}$. As has been discussed by Nalewajko et al. (2014b), the luminosity ratio of the Comptonization and synchrotron SED components $q=L_{\mathrm{IC}} / L_{\mathrm{syn}}$, referred to as the Compton dominance parameter, is an indirect probe of the jet magnetisation $q \simeq(\xi / 0.005)(\Gamma / 20)^{2}\left(\Gamma \theta_{\mathrm{j}}\right)^{2}\left(L_{\mathrm{d}} / L_{\mathrm{B}}\right)$, where $\xi$ is a parameter related to the covering factor of the medium reprocessing the accretion disk radiation of luminosity $L_{\mathrm{d}}$, and $L_{\mathrm{B}}$ is the magnetic jet power. Highly magnetised emitting regions in blazar jets are expected to produce radiation characterised by low Compton dominance $q \sim 1$ and high synchrotron luminosity $L_{\text {syn }}>10^{47} \mathrm{erg} \mathrm{s}^{-1}$ (Janiak et al. 2015). The eventual existence 
of such blazars would challenge the established theoretical expectation of rough equipartition in the blazar zone.

The SEDs of all types of blazars appear to be organised into so-called blazar sequence, the primary statement of which is an anticorrelation between the synchrotron SED peak frequency $v_{\text {syn }}$ and the bolometric synchrotron luminosity $L_{\text {syn }}$ (Fossati et al. 1998). The second statement is that the Compton dominance $q$ increases with increasing $L_{\mathrm{syn}}$, that is, that $L_{\mathrm{IC}}$ increases faster than $L_{\text {syn }}$. Hence, at the low-luminosity end of the sequence we have so-called high-frequency peaked BL Lac objects (HBL) with $v_{\text {syn }} \sim 10^{16} \mathrm{~Hz}, L_{\text {syn }} \sim 10^{44} \mathrm{erg} \mathrm{s}^{-1}$ and $q \sim 1$; and at the high-luminosity end of the sequence we have flat-spectrum radio quasars (FSRQ) with $v_{\text {syn }} \sim 10^{13} \mathrm{~Hz}$, $L_{\text {syn }} \sim 10^{47} \mathrm{erg} \mathrm{s}^{-1}$ and $q \sim 10-100$. Since the synchrotron peak frequency for FSRQs is expected in the far-infrared band, it is very difficult to observe it directly, and it has been mostly determined by fitting simple SED templates to the radio/mm and NIR/optical data.

A sensitive all-sky survey in the mid-infrared (MIR) band was performed by the WISE satellite in 2010 (Wright et al. 2010) and most gamma-ray bright blazars can be associated with a WISE source. Furthermore, it has been demonstrated that blazars occupy a narrow region in a MIR colour-colour diagram (Massaro et al. 2011), which has been very useful for identifying new blazar candidates. A combined WISE and Fermi-LAT sample of blazars was studied by D'Abrusco et al. (2012), who analysed the observed distributions of infrared and gamma-ray colours, spectral indices, fluxes, and the Compton dominance parameter.

Here, we perform further analysis of a combined Fermi-LAT and WISE sample of blazars with known redshifts. In Sect. 2 we describe the selection of our sample. In Sect. 3 we present the distributions of physical parameters of blazar SEDs (luminosities, photon indices and Compton dominance) derived from infrared-only and combined infrared/gamma-ray data for our sample. In Sect. 4 we present a very simple one-zone leptonic model of emission from relativistic jets, the predictions of which are compared directly with the observed distribution of synchrotron luminosity and Compton dominance. We conclude with a brief discussion in Sect. 5.

To calculate the apparent luminosities of blazars, we adopted a standard $\Lambda$ cold dark matter cosmology with $H_{0}=71 \mathrm{~km} \mathrm{~s}^{-1}$, $\Omega_{\mathrm{m}}=0.3$, and $\Omega_{\Lambda}=0.7$.

\section{WISE/Fermi blazar sample}

This study is based on the 2LAC catalogue of gamma-raybright blazars detected by Fermi-LAT between 2008 August and 2010 August (Ackermann et al. 2011) crossed with the AllWISE source catalogue of infrared point sources ${ }^{1}$. We have deliberately not used a more recent $3 \mathrm{LAC}$ catalogue based on 4 years of Fermi-LAT data (2008 August-2012 August; Ackermann et al. 2015), since the most sensitive cryogenic part of the WISE survey lasted from 2010 January to 2010 September, and hence the $2 \mathrm{LAC}$ results are more likely to be simultaneous with WISE observations.

From 2LAC, we took 310 flat-spectrum radio quasars (FSRQs) and 175 BL Lac objects (BL Lacs) with known spectroscopic redshifts and searched for their infrared counterparts in the WISE catalogue using the matching radius of 2 arcsec. This resulted in a sample of 152 FSRQs and 97 BL Lacs.

http://wise2.ipac.caltech.edu/docs/release/allwise/
WISE observed the Universe in four infrared bands with the following central wavelengths: $3.4 \mu \mathrm{m}(W 1), 4.6 \mu \mathrm{m}(W 2)$, $11.6 \mu \mathrm{m}(W 3)$, and $22.1 \mu \mathrm{m}(W 4)$. In order to convert the measured magnitudes to fluxes, we use the zero-point fluxes provided by Wright et al. (2010): $F_{W 1}=307 \mathrm{Jy}, F_{W 2}=171 \mathrm{Jy}$, $F_{W 3}=29 \mathrm{Jy}$, and $F_{W 4}=8.3 \mathrm{Jy}$.

\section{Observational results}

\subsection{Infrared properties}

Here we present results based solely on the WISE survey data. Our starting point is the WISE blazar strip established by (Massaro et al. 2011) using a colour-colour diagram $W_{1}-W_{2}$ vs. $W_{2}-W_{3}$. We translate this result into a photon index diagram $\Gamma_{12}$ versus $\Gamma_{23}$ (Fig. 1; left panel), using the standard definition of the photon index: $N(E) \propto E^{-\Gamma}$. In general, FSRQs are found to have soft infrared SEDs $(\Gamma>2)$ and BL Lacs have hard infrared SEDs $(\Gamma<2)$, which is in line with the blazar sequence (Fossati et al. 1998), and also with its latest revision including the WISE data (Ghisellini et al. 2017). As for the spectral curvature, we adopt a convention that a positive curvature corresponds to the natural shape of the synchrotron SED: $\log N(E)=C-\alpha \log E-\beta \log ^{2} E$. In the case of FSRQs, we find a preference for positive curvature with the mean value of $\Gamma_{12}-\Gamma_{23}=\beta \log \left(E_{1} / E_{3}\right)=0.36$, hence $\beta \sim 0.68$. However, in the case of BL Lacs, we find a negative curvature for $\Gamma_{12}<1.0$. Negative curvature suggests a superposition of two spectral components, most likely a contamination of the synchrotron component with radiation from the host galaxy.

If instead we look at the relation between photon indices $\Gamma_{23}$ versus $\Gamma_{34}$, for sources that are significantly detected $(S / N>10)$ in all $W_{2}, W_{3}, W_{4}$ bands, we find predominantly negative curvatures with $\Gamma_{34}>\Gamma_{23}$. This is highly suspicious, and we suspect that the $W_{4}$ fluxes may be systematically overestimated.

Even though the $W 4$ measurements are relatively poor, it is interesting to consider them as a possible probe of synchrotron self-absorption (SSA). The characteristic SSA frequency $v_{\mathrm{SSA}}$ is a sensitive probe of the radius $R$ of the emitting region, at the same time it depends only weakly on the jet Lorentz factor $\Gamma_{j}$ and the synchrotron luminosity $L_{\mathrm{syn}}$. If the emitting region is located at relatively large distance scales $(r \sim 1-10 \mathrm{pc})$, the SSA break can be located in the millimeter band (Sikora et al. 2008). However, in the case of very compact blazar-emitting regions, for example, located at distance scales within the broad-line region (BLR; $r<0.1 \mathrm{pc}$ ), the SSA break will be pushed to the MIR band (Hayashida et al. 2012). If the SSA break could coincide with the W3 band, we would expect a very hard spectrum at longer wavelengths $\left(\Gamma_{34}<1\right)$ with normal spectrum at shorter wavelengths $\left(\Gamma_{23} \simeq 2\right)$. Looking at Fig. 1 , we do not have any indication for this. On the other hand, several FSRQs are characterised by hard $\Gamma_{23}<1.5$ and normal $\Gamma_{12} \simeq 2-2.5$, which could be a signature of an SSA break located near the W2 band.

In Fig. 2, we show the distribution of photon index $\Gamma_{12}$ plotted against the MIR luminosity $L_{1}=\left(v L_{v}\right)_{W 1}$, calculated using spectroscopic redshifts provided in the $2 \mathrm{LAC}$ catalogue. We first note that the mid-infrared luminosities of blazars reach values up to $L_{1, \max } \sim 10^{47} \mathrm{erg} \mathrm{s}^{-1}$. The luminosities of FSRQs extend in the range $45<\log L_{1}<47$ and the luminosities of BL Lacs are in the range $44<\log L_{1}<46.5$. The most luminous BL Lacs $\left(\log L_{1}>45.5\right)$ overlap with the FSRQs and are characterised by relatively soft MIR spectra with $\Gamma_{12} \gtrsim 2$. Considering the entire sample of blazars, or BL Lacs alone, a trend of softerwhen-brighter is clearly seen (Pearson $=0.68)$, consistent with the blazar sequence. There are, however, significant outliers; for 

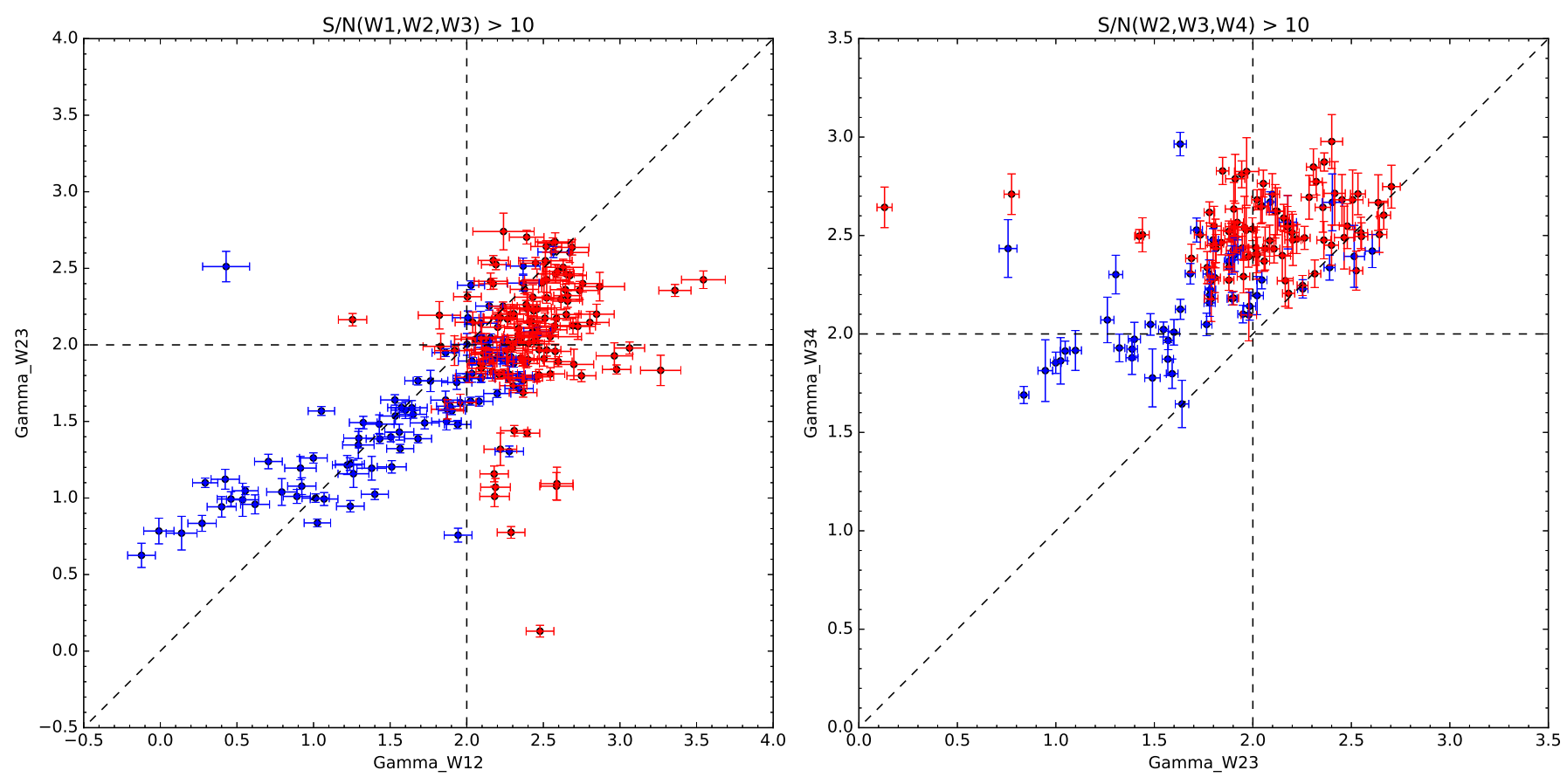

Fig. 1. Distribution of infrared photon index values of blazars determined from the WISE photometry. Left panel: $\Gamma_{W 23}$ vs. $\Gamma_{W 12}$. Right panel: $\Gamma_{W 34}$ vs. $\Gamma_{W 23}$. FSRQs (red) and BL Lacs (blue).

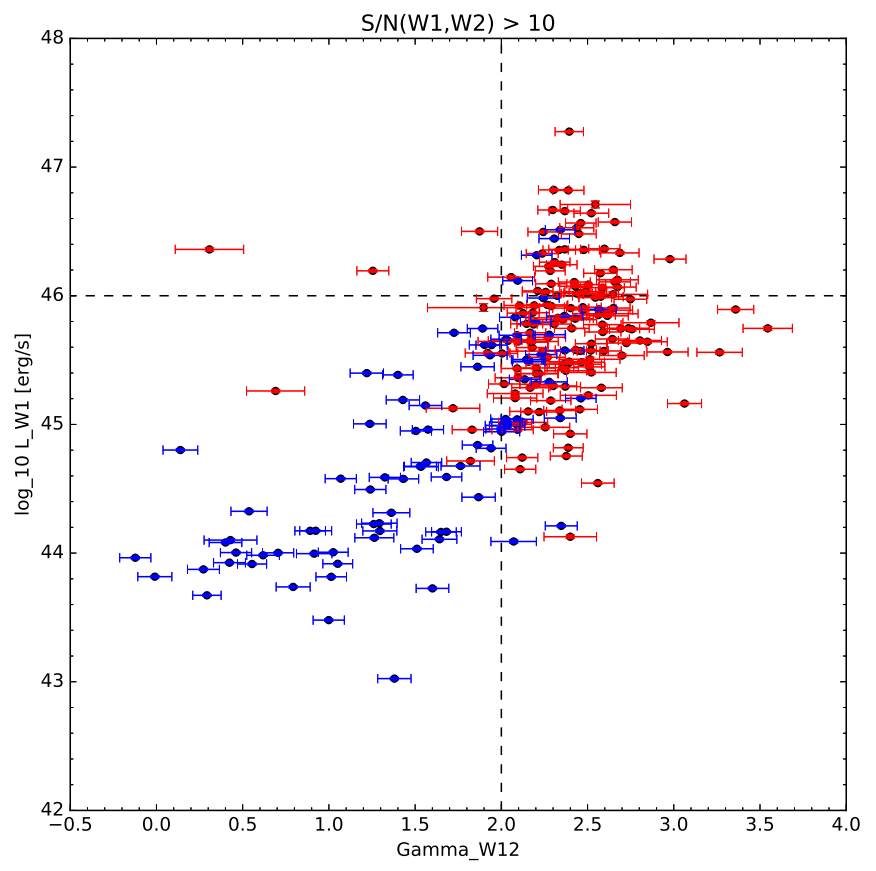

Fig. 2. Distribution of infrared luminosity $L_{W 1}$ vs. infrared photon index $\Gamma_{W 12}$ for blazars. FSRQs (red) and BL Lacs (blue).

example, a handful of luminous hard FSRQs with $\log L_{1}>46$ and $\Gamma_{12}<2$. These sources could be examples of luminous high-peaked blazars identified by Padovani et al. (2012). The hardest BL Lacs, those with $\Gamma_{12}<1$, appear to cluster around $\log L_{1} \sim 44$. As we noted previously, these sources are also characterised with negative spectral curvature with $\Gamma_{23}>\Gamma_{12}$, and hence they could actually be dominated by contribution from the host galaxy.
We compared our distribution of $L_{1}$ luminosities with the synchrotron SED peaks $L_{\text {syn,peak,F13 }}$ calculated by modelling the broad-band SEDs with two-component log-polynomial models (Finke 2013). With the spectral coverage of the entire infrared band being in general very poor, this method amounts to extrapolation of spectra measured in the radio/mm and NIR/optical/UV bands. We found that regardless of the order of magnitude for either luminosity estimate, the ratio $\log \left(L_{1} / L_{\text {syn,peak,F13 }}\right)$ has approximately uniform distribution in the range $[-1.2: 0]$. Hence, the extrapolated synchrotron peaks (their $v L_{v}$ rather than bolometric luminosities) exceed the observed MIR luminosities by up to one order of magnitude both for BL Lacs and FSRQs. The most likely reason for such discrepancy is that extrapolation of radio/mm or NIR/optical/UV spectra can be affected by additional spectral features, for example, a synchrotron selfabsorption break in the (sub)-mm band or the accretion disk continuum, especially at higher redshifts $(z \gtrsim 2)$.

\subsection{Infrared versus gamma-ray properties}

In Fig. 3, we show the distribution of gamma-ray photon index $\Gamma_{1-100 \mathrm{GeV}}$ plotted against the MIR photon index $\Gamma_{W 12}$ (cf. Fig. 6 in D'Abrusco et al. 2012). There is a clear correlation between the two indices (Pearson $=0.69$ ), although the range of $\Gamma_{1-100 \mathrm{GeV}} \in[1.5: 3]$ is more narrow. Most FSRQs are characterised both by soft MIR SED $\left(\Gamma_{W 12}>2\right)$ and soft gamma-ray SED $\left(\Gamma_{1-100 \mathrm{GeV}}>2\right)$. Most BL Lacs, excluding those that partially overlap with the FSRQs, are characterised by hard MIR SED $\left(\Gamma_{W 12}<2\right)$ and flat or moderately hard gamma-ray SED $\left(\Gamma_{1-100 \mathrm{GeV}} \lesssim 2\right)$. Those BL Lacs with the hardest MIR SED $\left(\Gamma_{W 12}<1\right)$, that are suspected to be contaminated by their host galaxies, are characterised by $\Gamma_{1-100 \mathrm{GeV}} \simeq 1.7$.

In Fig. 4, we show the distribution of the Compton dominance $q$ plotted against the MIR luminosity $L_{W 1}$. The Compton dominance is calculated as $q_{\mathrm{FW}}=L_{1 \mathrm{GeV}} / L_{W 1}$, where $L_{1 \mathrm{GeV}}$ is the $v L_{v}$ luminosity determined from the power-law fit over 


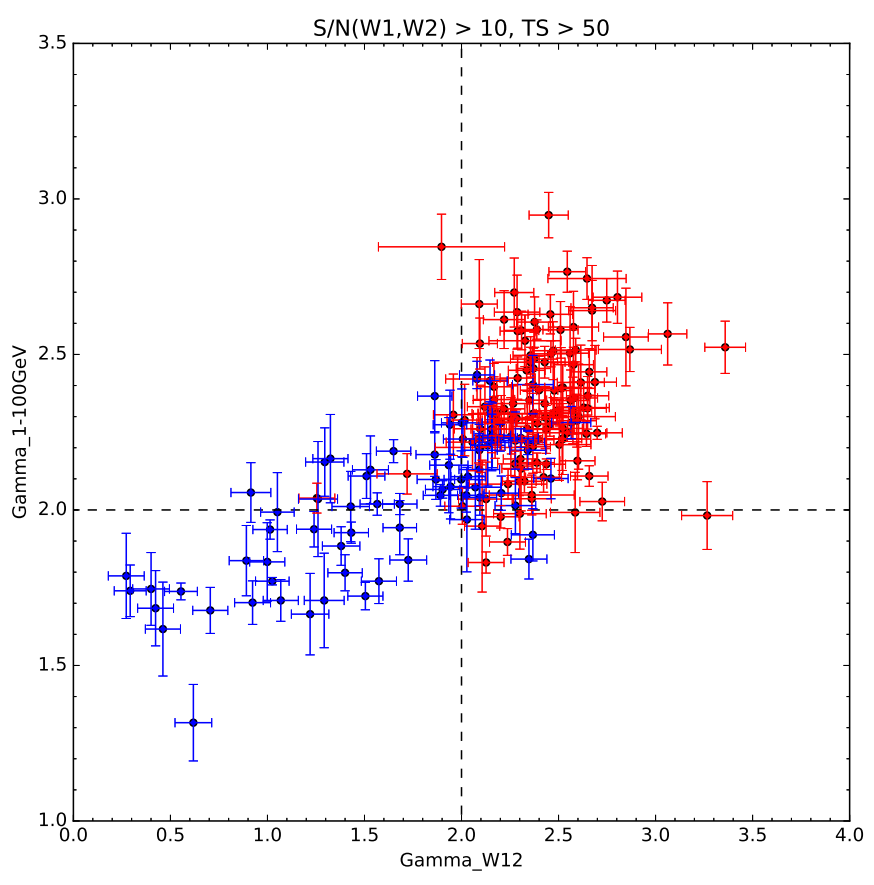

Fig. 3. Distribution of gamma-ray photon index $\Gamma_{1-100 \mathrm{GeV}}$ vs. infrared photon index $\Gamma_{W 12}$ for blazars. FSRQs (red) and BL Lacs (blue).

photon energy range $1-100 \mathrm{GeV}$ as provided in $2 \mathrm{LAC}$. We note that by using single-band luminosities $L_{W 1}$ and $L_{1 \mathrm{GeV}}$, we ignore bolometric corrections that can be up to one order of magnitude. However, for most blazars these corrections are expected to be comparable, so they should at least partially cancel out. We find that most FSRQs are characterised by $1<q_{\mathrm{FW}}<10$, with only a few reaching values $q_{\mathrm{FW}}>30$. On the other hand, most BL Lacs are characterised by $q_{\mathrm{FW}}<1$, with the observational lower limit of $q_{\mathrm{FW}}>0.1$. There is a substantial overlap of FSRQs and BL Lacs, with some FSRQs having $q_{\mathrm{FW}} \sim 0.3$ and some BL Lacs having $q_{\mathrm{FW}} \sim 5$. Considering the most synchrotron-luminous blazars with $L_{W 1}>10^{46} \mathrm{erg} / \mathrm{s}$, they span more than two orders of magnitude in Compton dominance with $0.3<q_{\mathrm{FW}}<50$. Sources with $L_{W 1}>10^{46} \mathrm{erg} / \mathrm{s}$ and $q_{\mathrm{FW}}<1$ are potentially most interesting as sites of efficient dissipation in highly magnetised jet regions (Janiak et al. 2015). In the following section, we discuss a simple model of blazar emission from a relativistic jet that allows to one to calculate the luminosities of the synchrotron and inverse Compton components of the SED.

Figure 5 shows the distribution of Compton dominance $q_{\mathrm{FW}}$ plotted against the MIR photon index $\Gamma_{W 12}$ or the gamma-ray photon index $\Gamma_{1-100 \mathrm{GeV}}$ (cf. Fig. 9 in D'Abrusco et al. 2012). The separation between FSRQs and BL Lacs appears to be cleaner in the $\Gamma_{W 12}$ versus $q_{\mathrm{FW}}$ space: most FSRQs are grouped in the region where $\Gamma_{W 12} \in[2: 3)$ and $\log _{10} q_{\mathrm{FW}} \in[0: 1.5)$, while most BL Lacs are found in the region where $\Gamma_{W 12} \in(0: 2]$ and $\log _{10} q_{\mathrm{FW}} \in(-1: 0]$. A correlation between $\Gamma_{1-100 \mathrm{GeV}}$ and $q_{\mathrm{FW}}$ is rather weak $($ Pearson $=0.26)$.

\section{Model of jet energetics}

We consider a simple one-zone leptonic model of blazar emission that can most naturally explain the distribution of apparent luminosities and Compton dominance along the blazar sequence. While this has been discussed in several previous studies (Meyer et al. 2011; Finke 2013), here we are guided by the following questions: (1) could blazar sequence be governed by the jet power? (2) What constraints can be put on the jet composition, that is, the relation between magnetic and leptonic jet powers? (3) What is the role of the bulk Lorentz factor along the sequence?

We consider a jet of conical geometry with the bulk velocity $\beta_{\mathrm{j}}=v_{\mathrm{j}} / c$, the corresponding bulk Lorentz factor $\Gamma_{\mathrm{j}}=$ $\left(1-\beta_{\mathrm{j}}^{2}\right)^{-1 / 2}$, and the opening angle $\theta_{\mathrm{j}}$. The emitting region is fixed in the external frame and is approximated as a cylindrical shell with radius $R$ and length $\Delta r_{\mathrm{em}}$. The shell radius is equal to the jet radius $R=r_{\text {diss }} \theta_{\mathrm{j}}$ at the distance scale $r_{\text {diss }}$, and the length $\Delta r_{\mathrm{em}}=\Gamma_{\mathrm{j}} c \tau_{\text {cool }}^{\prime}$ is related to the co-moving cooling time scale $\tau_{\text {cool }}^{\prime}$. We consider a population of relativistic electrons (or positrons) of co-moving number density $n_{\mathrm{e}}^{\prime}$ and mean Lorentz factor $\gamma_{\mathrm{e}}^{\prime}$, and their sub-population of emitting electrons of density $n_{\mathrm{em}}^{\prime}<n_{\mathrm{e}}^{\prime}$ and typical Lorentz factor $\gamma_{\mathrm{em}}^{\prime}$ such that $\gamma_{\mathrm{e}}^{\prime}<\gamma_{\mathrm{em}}^{\prime}<\left(n_{\mathrm{e}}^{\prime} / n_{\mathrm{em}}^{\prime}\right) \gamma_{\mathrm{e}}^{\prime}$. The population of emitting electrons produces radiation isotropic in the co-moving frame with emission power per particle $P_{\mathrm{em}, 1}^{\prime}$ such that its radiative cooling time scale is $\tau_{\text {cool }}^{\prime}=\gamma_{\mathrm{em}} m_{\mathrm{e}} c^{2} / P_{\mathrm{em}, 1}^{\prime}$. Transforming the total emission power $P_{\mathrm{em}}^{\prime}=n_{\mathrm{em}}^{\prime} V_{\mathrm{em}}^{\prime} P_{\mathrm{em}, 1}^{\prime}$ into the external frame, we note that (1) the effective length of the emitting region in the co-moving frame is $\Delta r_{\mathrm{em}}^{\prime}=\Delta r_{\mathrm{em}} / \Gamma_{\mathrm{j}}=c \tau_{\text {cool }}^{\prime}$, (2) hence the volume transformation is $V_{\mathrm{em}}^{\prime}=\pi R^{2} \Delta r_{\mathrm{em}}^{\prime}=V_{\mathrm{em}} / \Gamma_{\mathrm{j}}$, (3) the number density of electrons transforms like $n_{\mathrm{e}(\mathrm{em})}=\Gamma_{\mathrm{j}} n_{\mathrm{e}(\mathrm{em})}^{\prime}$, and hence (4) the effective number of emitting electrons is $N_{\mathrm{em}}=n_{\mathrm{em}} V_{\mathrm{em}}=\Gamma_{\mathrm{j}}^{2} N_{\mathrm{em}}^{\prime}$, (5) the power emitted by each electron is invariant $P_{\mathrm{em}, 1}=P_{\mathrm{em}, 1}^{\prime}$, and thus (6) the result is $P_{\mathrm{em}}=\Gamma_{\mathrm{j}}^{2} P_{\mathrm{em}}^{\prime}$. This can be compared with the total electron energy flux $P_{\mathrm{e}}=\pi R^{2} \Gamma_{\mathrm{j}}^{2} \gamma_{\mathrm{e}}^{\prime} n_{\mathrm{e}}^{\prime} m_{\mathrm{e}} c^{3}$, and we find that $P_{\mathrm{em}} / P_{\mathrm{e}}=\left(\gamma_{\mathrm{em}} / \gamma_{\mathrm{e}}\right)\left(n_{\mathrm{em}}^{\prime} / n_{\mathrm{e}}^{\prime}\right) \equiv \epsilon_{\mathrm{em}}$, which we call the radiation efficiency of jet electrons.

The emitted radiation in the external frame is strongly anisotropic due to (1) the relativistic Doppler effect on photon energy $E_{\gamma}=\mathcal{D} E_{\gamma}^{\prime}$, and due to (2) the relativistic aberration affecting the solid angle $\mathrm{d} \Omega=\mathrm{d} \Omega^{\prime} / \mathcal{D}^{2}$ or the cosine of the viewing angle $\mu_{\text {obs }}=\cos \theta_{\text {obs }}$ so that $\mathrm{d} \mu_{\text {obs }}=\mathrm{d} \mu_{\text {obs }}^{\prime} / \mathcal{D}^{2}$, where $\mathcal{D}=$ $\left[\Gamma_{\mathrm{j}}\left(1-\beta_{\mathrm{j}} \mu_{\mathrm{obs}}\right)\right]^{-1}=\Gamma_{\mathrm{j}}\left(1+\beta_{\mathrm{j}} \mu_{\mathrm{obs}}^{\prime}\right)$ is the relativistic Doppler factor. The apparent luminosity is $L_{\mathrm{obs}}\left(\mu_{\mathrm{obs}}\right)=\left(\mathcal{D}^{3} / \Gamma_{\mathrm{j}}\right) P_{\mathrm{em}}=\mathcal{D}^{3} \Gamma_{\mathrm{j}} P_{\mathrm{em}}^{\prime}$, so that $\left\langle L_{\mathrm{obs}}\right\rangle_{\Omega}=P_{\mathrm{em}}$ (Sikora et al. 1997; Jester 2008).

We now consider three main leptonic radiative mechanisms that are relevant for blazars: synchrotron, synchrotron selfCompton (SSC), and external radiation Comptonization (ERC). For either of them, the emission power of a single electron is $P_{\mathrm{em}, 1}^{\prime}=(4 / 3) \sigma_{\mathrm{T}} c \gamma_{\mathrm{em}}^{2} u_{0}^{\prime}$, where $u_{0}^{\prime}$ stands for magnetic energy density $u_{\mathrm{B}}^{\prime}=B^{\prime 2} /(8 \pi)$ in the case of synchrotron, energy density of synchrotron radiation $u_{\text {syn }}^{\prime}$ in the case of SSC, and external radiation density $u_{\text {ext }}^{\prime}$ in the case of ERC. We can relate the magnetic energy density to the magnetic jet power $P_{\mathrm{B}}=\pi R^{2} \Gamma_{\mathrm{j}}^{2} u_{\mathrm{B}}^{\prime} c$, and the external radiation energy density can be expressed as $u_{\text {ext }}^{\prime} \simeq \mathcal{D}^{2} L_{\text {ext }} /\left(4 \pi c r_{\text {ext }}^{2}\right)$ (Dermer 1995), where $L_{\text {ext }}$ is the luminosity of external radiation sources or characteristic radius $r_{\text {ext }} \sim r_{\text {diss }}$. The co-moving energy density of synchrotron radiation is approximately $u_{\mathrm{syn}}^{\prime} \simeq g_{\mathrm{ssc}} P_{\mathrm{syn}}^{\prime} /\left(4 \pi c R^{2}\right)$, where $P_{\text {syn }}^{\prime}=P_{\text {syn, } 1}^{\prime} n_{\mathrm{em}}^{\prime} V_{\mathrm{em}}^{\prime}$ and $g_{\mathrm{ssc}}$ is a geometric factor. The value of $g_{\text {ssc }}$ depends on the shape (mainly the aspect ratio) of the synchrotron-emitting region in the co-moving frame, light travel effects and anisotropy of IC scattering; it is therefore difficult to calculate and relatively uncertain. The effective co-moving radiative cooling time scale is now $\tau_{\text {cool }}^{\prime}=3 m_{\mathrm{e}} c /\left(4 \sigma_{\mathrm{T}} \gamma_{\mathrm{em}} u_{\mathrm{tot}}^{\prime}\right)$, where $u_{\mathrm{tot}}^{\prime}=u_{\mathrm{B}}^{\prime}+u_{\mathrm{syn}}^{\prime}+u_{\mathrm{ext}}^{\prime}$, and the respective luminosities 

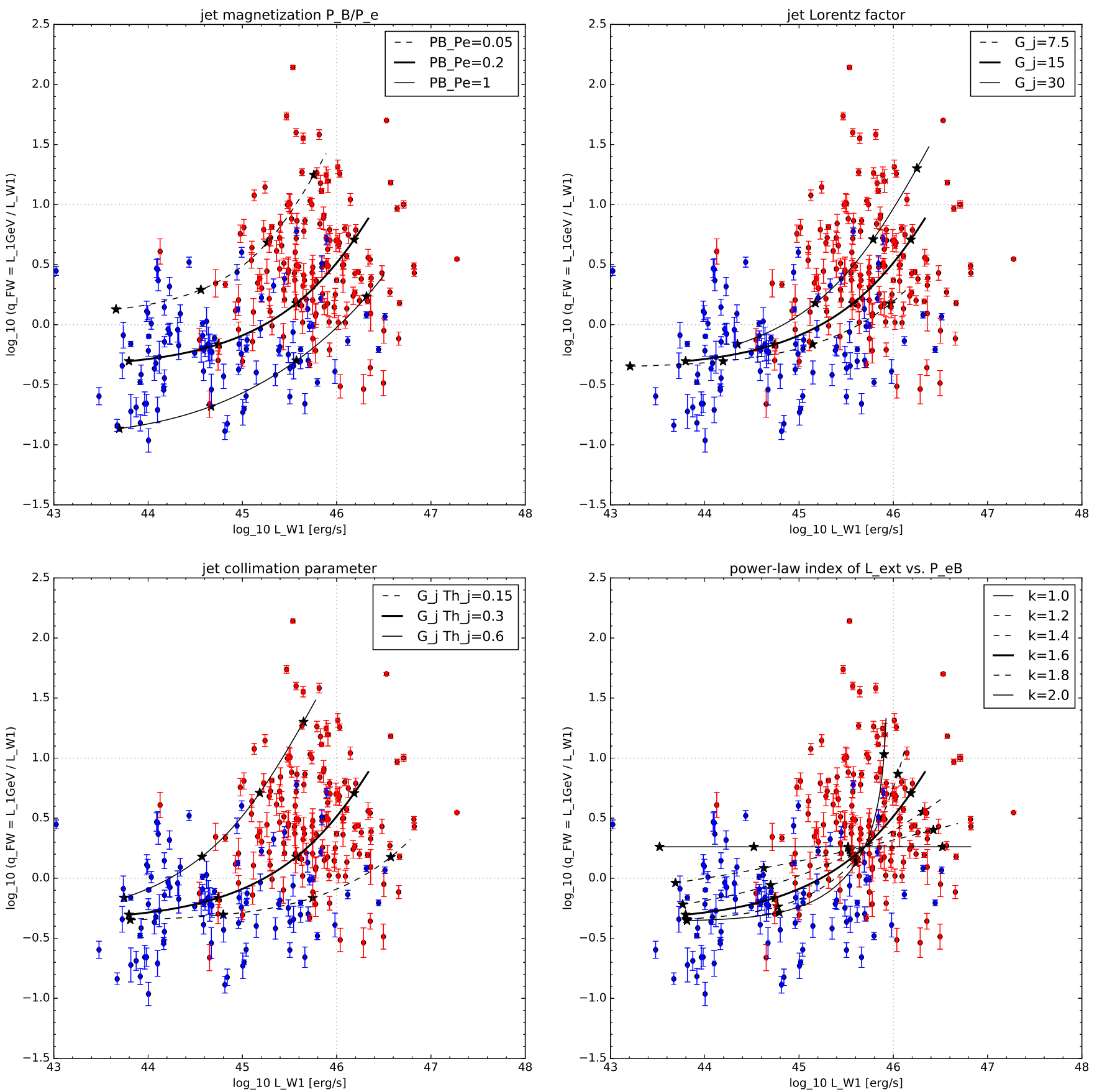

Fig. 4. Distribution of Fermi + WISE blazars in the parameter space of Compton dominance $q_{\mathrm{FW}}=L_{\gamma} / L_{\mathrm{syn}}$ and synchrotron luminosity $L_{\mathrm{syn}}$, where $L_{\gamma}=L_{\mathrm{SSC}}+L_{\mathrm{ERC}}\left(\right.$ FSRQs - red, BL Lacs - blue). Thick solid lines show our reference model of the blazar sequence for $\Gamma_{\mathrm{j}}=15, P_{\mathrm{B}} / P_{\mathrm{e}}=0.2$, $\Gamma_{\mathrm{j}} \theta_{\mathrm{j}}=0.3, \epsilon_{\mathrm{em}}=0.5$, and $\kappa=1.6$. Top left panel: dependence of the model on the jet magnetisation $P_{\mathrm{B}} / P_{\mathrm{e}}=0.05$ (dashed), 1 (thin solid). Top right panel: dependence of the model on the jet Lorentz factor $\Gamma_{\mathrm{j}}=7.5$ (dashed), 30 (thin solid). Bottom left panel: dependence of the model on the jet collimation factor $\Gamma_{\mathrm{j}} \theta_{\mathrm{j}}=0.15$ (dashed), 0.6 (thin solid). Bottom right panel: dependence of the model on the power-law index $\kappa$ in the scaling of external radiation luminosity $L_{\mathrm{ext}} \propto P_{\mathrm{eB}}^{\kappa}$. The black stars along each track indicate the lepto-magnetic jet power values $\log _{10} P_{\mathrm{eB}}=42,43,44,45$.

are $L_{\mathrm{syn}}=\left(u_{\mathrm{B}}^{\prime} / u_{\mathrm{tot}}^{\prime}\right) L_{\mathrm{obs}}, L_{\mathrm{SSC}}=\left(u_{\mathrm{syn}}^{\prime} / u_{\mathrm{tot}}^{\prime}\right) L_{\mathrm{obs}}$, and $L_{\mathrm{ERC}}=$ $\left(u_{\mathrm{ext}}^{\prime} / u_{\mathrm{tot}}^{\prime}\right) L_{\mathrm{obs}}$.

We now consider two components of the Compton dominance parameter:

$$
\begin{aligned}
& q_{\mathrm{SSC}}=\frac{L_{\mathrm{SSC}}}{L_{\mathrm{syn}}}=\frac{u_{\mathrm{syn}}^{\prime}}{u_{\mathrm{B}}^{\prime}}=\frac{g_{\mathrm{ssc}} \epsilon_{\mathrm{em}}}{4} \frac{L_{\mathrm{syn}}}{L_{\mathrm{obs}}} \frac{P_{\mathrm{e}}}{P_{\mathrm{B}}}, \\
& q_{\mathrm{ERC}}=\frac{L_{\mathrm{ERC}}}{L_{\mathrm{syn}}}=\frac{u_{\mathrm{ext}}^{\prime}}{u_{\mathrm{B}}^{\prime}}=\left(\frac{\mathcal{D} \Gamma_{\mathrm{j}} \theta_{\mathrm{j}}}{2}\right)^{2} \frac{L_{\mathrm{ext}}}{P_{\mathrm{B}}}
\end{aligned}
$$

In order to explain the observational trend of Compton dominance $q_{\mathrm{FW}}$ increasing systematically over three orders of magnitude in $L_{\mathrm{syn}}$, these functions should somehow scale with the lepto-magnetic jet power $P_{\mathrm{eB}}=P_{\mathrm{e}}+P_{\mathrm{B}}$ (this can be generalised by including the contribution from protons) or with the bulk Lorentz factor $\Gamma_{\mathrm{j}}$. As is typical, we assume that $\mathcal{D} \propto \Gamma_{\mathrm{j}}$, $\theta_{\mathrm{j}} \propto 1 / \Gamma_{\mathrm{j}}, R \propto r_{\mathrm{diss}} / \Gamma_{\mathrm{j}}$. We also assume that $\epsilon_{\mathrm{em}}$ is comparable for FSRQs and BL Lacs.

When the total emission is dominated by synchrotron (and SSC; $L_{\text {obs }} \sim L_{\text {syn }} \gtrsim L_{\mathrm{SSC}} \gg L_{\mathrm{ERC}}$ ), as in the case of BL Lacs, 

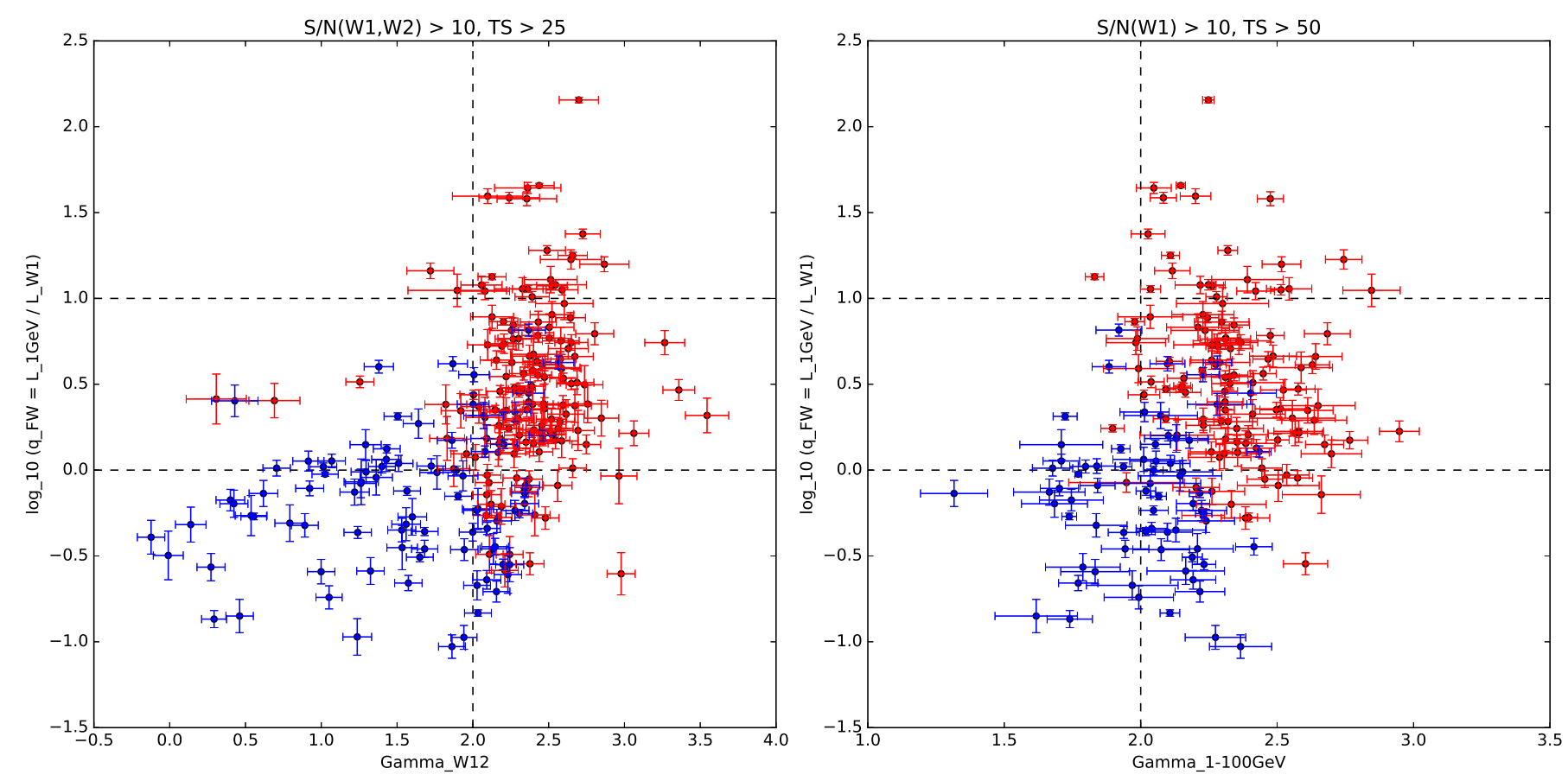

Fig. 5. Distribution of Compton dominance $q_{\mathrm{FW}}=L_{1-100 \mathrm{GeV}} / L_{W 1}$ vs. infrared photon index $\Gamma_{W 12}$ (left panel) and gamma-ray photon index $\Gamma_{1-100 \mathrm{GeV}}$ (right panel) for blazars. FSRQs (red) and BL Lacs (blue).

$q_{\mathrm{SSC}} \propto P_{\mathrm{e}} / P_{\mathrm{B}}$. On the other hand, when the total emission is dominated by $\operatorname{ERC}\left(L_{\mathrm{obs}} \sim L_{\mathrm{ERC}} \gg \max \left(L_{\mathrm{syn}}, L_{\mathrm{SSC}}\right)\right)$, as in the case of FSRQs, we find that $q_{\mathrm{SSC}} \propto P_{\mathrm{e}} /\left(q_{\mathrm{ERC}} P_{\mathrm{B}}\right)$. Hence, the energy balance between electrons and magnetic fields, that is, the equipartition condition, is key in determining the value of $q_{\mathrm{SSC}}$.

The value of $q_{\text {ERC }}$ depends primarily on the relation between external radiation luminosity $L_{\text {ext }}$ and the magnetic jet power $P_{\mathrm{B}}$. It is typically assumed that external radiation fields result from reprocessing of accretion flow luminosity $L_{\text {acc }}$ by external medium (whether the broad line region or the dusty torus) of covering factor $\xi_{\text {ext }} \sim 0.1-0.3$ (e.g. Calderone et al. 2012), such that $L_{\text {ext }}=\xi_{\text {ext }} L_{\text {acc }}$. The accretion luminosity is related to the accretion power $P_{\text {acc }} \equiv \dot{M}_{\text {acc }} c^{2}$ via radiative efficiency $\epsilon_{\text {acc }}$, such that $L_{\mathrm{acc}}=\epsilon_{\mathrm{acc}} P_{\mathrm{acc}}$. Further, the accretion power can be related to the lepto-magnetic jet power by a parameter called jet production efficiency $\eta_{\mathrm{eB}}=P_{\mathrm{eB}} / P_{\mathrm{acc}}$. While we cannot directly estimate the value of $P_{\mathrm{acc}}$, we can constrain the combination of parameters $\xi_{\text {ext }} \epsilon_{\text {acc }} / \eta_{\mathrm{eB}}$ by postulating a systematic relation between luminosity of external radiation and the total jet power $L_{\mathrm{ext}}=A\left(P_{\mathrm{eB}} / P_{\mathrm{Edd} *}\right)^{\kappa}$, where $P_{\mathrm{Edd} *}=1.5 \times 10^{47} \mathrm{erg} \mathrm{s}^{-1}$ is the Eddington luminosity for a reference value of a supermassive black hole of mass $M_{\mathrm{bh} *}=10^{9} M_{\odot}{ }^{2}$. The value of $\kappa$ can be in general constrained to be within the range $1<\kappa<2$. If $\kappa=1$, we would predict that $q_{\mathrm{ERC}}$ is independent of the jet power. On the other hand, if $\kappa=2$, the value of $q_{\text {ERC }}$ would become independent of $L_{\text {syn }}$.

Using this scheme, we seek a reference model of blazar sequence, in which most parameters are tied to the lepto-magnetic jet power $P_{\mathrm{eB}}$. For all models presented here, we consider the range $42<\log _{10} P_{\mathrm{eB}}<45$. For the reference model, we adopt the following key parameters: jet Lorentz factor $\Gamma_{j}=15$, jet opening angle $\theta_{\mathrm{j}}=0.3 / \Gamma_{\mathrm{j}}$, and radiative efficiency $\epsilon_{\mathrm{em}}=0.5$.

\footnotetext{
2 In fact, the masses of supermassive black holes in blazars span roughly two orders of magnitude, with $M_{\mathrm{bh}} / M_{\odot} \sim 5 \times 10^{7}-5 \times 10^{9}$ (Ghisellini \& Tavecchio 2015).
}

First, in order to match the observed distribution of Compton dominance values for BL Lacs, that is, the $q_{\mathrm{SSC}}$, we adjust the jet magnetisation described by the $P_{\mathrm{B}} / P_{\mathrm{e}}$ ratio. We have seen that $P_{\mathrm{B}} / P_{\mathrm{e}}$ affects $q_{\mathrm{SSC}}$, whether cooling is dominated by synchrotron or ERC. Moreover, if we limit ourselves to the case where $P_{\mathrm{B}}<P_{\mathrm{e}}$, since $L_{\mathrm{ext}}$ is scaled with $P_{\mathrm{eB}}=P_{\mathrm{B}}+P_{\mathrm{e}} \sim P_{\mathrm{e}}$, the value of $q_{\mathrm{ERC}}$ is also affected. We find that jet magnetisation in the range $0.05<P_{\mathrm{B}} / P_{\mathrm{e}}<1$ can explain the observed values of $q_{\mathrm{SSC}}$ most naturally. Hence, we adopt a reference value of $P_{\mathrm{B}} / P_{\mathrm{e}}=0.2$.

Second, in order to match the observed distribution of $q_{\mathrm{ERC}}$, we normalise the luminosity of external radiation as $L_{\text {ext }} \sim 5 \times$ $10^{47}\left(P_{\mathrm{eB}} / P_{\mathrm{Edd} *}\right)^{1.6} \mathrm{erg} \mathrm{s}^{-1}$, and hence we adopt $A=3.3 P_{\text {Edd } *}$ and $\kappa=1.6$. In the bottom right panel of Fig. 4 , we show the sensitivity of our reference model to the value of $\kappa$. We consider that the adopted value of $\kappa$ is accurate within $\sim 0.2$. Our reference model is now defined completely, and it is shown on every panel of Fig. 4 with thick solid lines.

Next, we consider the effects of jet Lorentz factor $\Gamma_{j}$ and the jet collimation parameter $\Gamma_{\mathrm{j}} \theta_{\mathrm{j}}$. In the top right panel of Fig. 4, we show models obtained from the reference models by setting $\Gamma_{\mathrm{j}}=7.5,30$, keeping fixed $\Gamma_{\mathrm{j}} \theta_{\mathrm{j}}=0.3$. On the other hand, in the bottom left panel of Fig. 4, we show models corresponding to $\Gamma_{\mathrm{j}} \theta_{\mathrm{j}}=0.15,0.6$, keeping fixed $\Gamma_{\mathrm{j}}=15$. We note that in the case of FSRQs, where the total radiation output is dominated by ERC, the synchrotron luminosity scales like $L_{\mathrm{syn}} \propto\left(\mathcal{D} / \Gamma_{\mathrm{j}}\right)\left(\Gamma_{\mathrm{j}} \theta_{\mathrm{j}}\right)^{-2}$, and Compton dominance scales like $q_{\text {ERC }} \propto\left(\mathcal{D} \Gamma_{\mathrm{j}} \theta_{\mathrm{j}}\right)^{2}$. Hence, the effect of jet collimation parameter is stronger, as it affects both $L_{\text {syn }}$ and $q_{\text {ERC. }}$. Adopting a larger value of $\Gamma_{\mathrm{j}} \theta_{\mathrm{j}}$ allows to achieve higher Compton dominance for the same value of $P_{\mathrm{B}} / P_{\mathrm{e}}$, or to achieve the same value of $q_{\mathrm{ERC}}$ for higher values of $P_{\mathrm{B}} / P_{\mathrm{e}}$.

\section{Discussion}

We considered a simple parametrisation of blazar emission models in order to understand the observed distribution of Compton 
dominance along the blazar sequence. In our model, most parameters are tied to the lepto-magnetic jet power $P_{\mathrm{eB}}$, the value of which extends over three orders of magnitude to reproduce the observed range of synchrotron luminosities $L_{\text {syn }}$. Theoretical Compton dominance has two components: $q_{\mathrm{SSC}}=L_{\mathrm{SSC}} / L_{\mathrm{syn}}$ and $q_{\mathrm{ERC}}=L_{\mathrm{ERC}} / L_{\mathrm{syn}}$, and under most plausible circumstances they both scale with the equipartition parameter $P_{\mathrm{B}} / P_{\mathrm{e}}$. In our reference model with $\Gamma_{\mathrm{j}}=15, \Gamma_{\mathrm{j}} \theta_{\mathrm{j}}=0.3, \epsilon_{\mathrm{em}}=0.5$, and $L_{\text {ext }} / P_{\text {Edd* }} \sim 3.3\left(P_{\mathrm{eB}} / P_{\mathrm{Edd} *}\right)^{1.6}$, the observed distribution of $L_{\gamma} / L_{\text {syn }}$ can be reproduced for $0.05<P_{\mathrm{B}} / P_{\mathrm{e}}<1$, which means a jet dominated by matter (even without counting protons). A very similar conclusion for the case of BL Lacs was obtained recently by Tavecchio \& Ghisellini (2016). Our reference model also implies typical values for the lepto-magnetic jet power: $\log _{10} P_{\mathrm{eB}} \sim 42-43.5$ in the case of BL Lacs, and $\log _{10} P_{\mathrm{eB}} \sim$ 43.5-45 in the case of FSRQs. The highest gamma-ray luminosities achieved by the FSRQs, $L_{1 \mathrm{GeV} \text {,max }} \sim 10^{48} \mathrm{erg} \mathrm{s}^{-1}$ (longterm average), imply the existence of an upper limit on the lepto-magnetic power of radiatively efficient relativistic AGN jets of $P_{\mathrm{eB}, \max } \sim 2 \times 10^{45} \mathrm{erg} \mathrm{s}^{-1} \simeq P_{\mathrm{Edd} *} / 75$, leaving plenty of room for protons. On the other hand, the highest observed synchrotron luminosities are systematically lower, with $L_{W 1, \text { max }} \sim$ $10^{47} \mathrm{erg} \mathrm{s}^{-1} \sim L_{1 \mathrm{GeV} \text {,max }} / 10$, much lower than values predicted for powerful jets under magnetisation $\sigma \sim 1$ (Janiak et al. 2015).

The existence of an upper limit on the jet power allows us to interpret the scaling of external radiation luminosity with the total jet power $L_{\mathrm{ext}} / P_{\mathrm{Edd} *} \sim 3.3\left(P_{\mathrm{eB}} / P_{\mathrm{Edd} *}\right)^{1.6}$. Assuming that $\xi_{\mathrm{ext}}, \eta_{\mathrm{eB}}=$ const, and that $\epsilon_{\mathrm{acc}}=\epsilon_{\mathrm{acc}, 0}\left(P_{\mathrm{acc}} / P_{\mathrm{acc}, \max }\right)^{0.6}$, where $P_{\mathrm{acc}, \max }=P_{\mathrm{eB}, \max } / \eta_{\mathrm{eB}}$, we find that $L_{\mathrm{acc}} / P_{\mathrm{Edd} *} \simeq$ $0.03 \xi_{\text {ext }-1}^{-1}\left(P_{\text {acc }} / P_{\text {acc, } \max }\right)^{1.6}$ and that $\xi_{\text {ext }} \epsilon_{\mathrm{acc}, 0} / \eta_{\mathrm{eB}} \simeq 0.25$. This shows that for the most powerful blazars with $P_{\mathrm{eB}} \sim P_{\mathrm{eB} \text {,max }}$, the requirements for radiative efficiency of accretion and the covering factor of radiation reprocessing medium are very tight.

Degeneracy of parameters is widely recognised as the main obstacle in constraining the physical parameters of relativistic blazar jets. The SSC branch of Compton dominance $q_{\mathrm{SSC}}$ can be affected by radiative efficiency of jet electrons $\epsilon_{\mathrm{em}}$, while the ERC branch depends sensitively on the jet Lorentz factor $\Gamma_{\mathrm{j}}$, the jet collimation parameter $\Gamma_{\mathrm{j}} \theta_{\mathrm{j}}$, and on the scaling of external radiation luminosity $L_{\text {ext }}$. However, the relatively limited range of observed values of Compton dominance, factor $\sim 100$ in the case of $q_{\mathrm{ERC}}$ and factor $\sim 30$ in the case of $q_{\mathrm{SSC}}$, means that there should be strict limits on the combinations of parameters, such as $\left(\mathcal{D} \Gamma_{\mathrm{j}} \theta_{\mathrm{j}}\right)^{2}\left(L_{\mathrm{ext}} / P_{\mathrm{B}}\right)$. This also means that while equipartition can be realised for FSRQs (e.g. Dermer et al. 2014) by increasing $\Gamma_{\mathrm{j}}$ or $\Gamma_{\mathrm{j}} \theta_{\mathrm{j}}$, it would be difficult to obtain for BL Lacs, as the adopted value of electron radiative efficiency $\epsilon_{\mathrm{em}}=50 \%$ is already high. Nevertheless, it has been suggested that magnetisations in blazar jets could be very high, with $P_{\mathrm{B}} \gg P_{\mathrm{e}}$, if the overall radiative efficiency (including magnetic fields but not including protons) is as high as $95 \%$ (Potter 2017). Also, the value of geometric factor $g_{\mathrm{SSC}}$ is relatively uncertain. High values of $g_{\mathrm{SSC}}$ could be obtained if the synchrotron emitting region is relatively thin compared with the jet radius $R$. This is the usual assumption of numerical models of blazar emission (Moderski et al. 2003), and hence such models are essential to constrain the value of $g_{\mathrm{SSC}}$.

Nevertheless, our very simplified model suggests that it is probably easier and more natural to explain the observed distribution of blazar SEDs as a function of $P_{\mathrm{eB}}, P_{\mathrm{B}} / P_{\mathrm{e}}$ for a single value of $\Gamma_{\mathrm{j}} \simeq 15$ (as suggested by Ghisellini et al. 2014 for the case of FSRQs), rather than a function of $P_{\mathrm{eB}}, \Gamma_{\mathrm{j}}$ for a single value of $P_{\mathrm{B}} / P_{\mathrm{e}} \simeq 0.2$. In the former scenario, the transition between SSC and ERC domination is roughly at the same value of $P_{\mathrm{eB}}$, while in the latter scenario some BL Lacs are predicted to be contaminated by ERC at high values of $\Gamma_{j}$. On the other hand, interferometric radio observations of blazars indicate that FSRQs have systematically higher jet Lorentz factors than BL Lacs (e.g. Hovatta et al. 2009).

Regarding the location of the emitting region (see Nalewajko et al. 2014a, for more details in the case of FSRQs), we assumed that the energy density of external radiation fields scales like $u_{\mathrm{ext}}^{\prime}=\Gamma_{\mathrm{j}}^{2} L_{\text {ext }} /\left(4 \pi c r_{\text {diss }}^{2}\right)$, a simple power-law dependence on distance scale $r_{\text {diss }}$. In reality, we can expect several contributions to the external photon fields with certain characteristic distance scales; for example, $r_{\mathrm{BLR}} \sim 0.1 L_{\mathrm{acc}, 46}^{1 / 2} \mathrm{pc}$ for broad emission lines or $r_{\mathrm{HDR}} \sim 3 L_{\mathrm{acc}, 46}^{1 / 2} \mathrm{pc}$ for the hot-dust region (torus). Also, effects of special relativity can modify the inversesquare law for radiation density. Nevertheless, the inverse-square law applies roughly over several orders of magnitude in $r_{\text {diss }}$, since in quasars the external radiation fields originate mostly from reprocessing of direct radiation of the innermost accretion disk (Sikora et al. 2009).

Of course, the observed distribution of Compton dominance values could be subject to various selection effects (Giommi et al. 2012; Finke 2013). On one hand, highly Compton-dominated sources could exist as unidentified extragalactic gamma-ray sources (e.g. Massaro et al. 2017). On the other hand, highly synchrotron-dominated sources could exist as bright optical/radio blazars not detected by Fermi-LAT (e.g. Lister et al. 2015). We should also note that comparing singleband observed luminosities ( $W 1$ and $1 \mathrm{GeV}$ ) with the bolometric luminosities predicted by our model ignores the bolometric correction $L_{\text {syn }} / L_{W 1}$, and the combination of bolometric corrections that affect Compton dominance $q_{\mathrm{FW}}$. Taking this into account, we can expect that the total jet powers predicted by our reference model could be underestimated by up to one order of magnitude.

The results of our simplified analysis suggest that the emitting regions of blazar jets are out of equipartition, with $P_{\mathrm{B}} / P_{\mathrm{e}} \sim 0.05-1$. This conclusion is more solid in the case of BL Lacs, since $q_{\mathrm{SSC}}$ does not depend directly on the jet Lorentz factor or opening angle. It is encouraging that this conclusion is confirmed by results of SED modelling for BL Lacs (Tavecchio \& Ghisellini 2016). That this conclusion should apply equally to FSRQs is motivated mainly by our implicit assumption that the composition of all blazar jets should be comparable. In our picture, the transition between BL Lacs and FSRQs can be explained solely due to systematic, faster-thanlinear increase of the luminosity of external radiation fields with increasing accretion rate. Should our conclusion hold, it has strong implications for the dissipation physics of relativistic blazar jets: dissipation models based on magnetic reconnection in magnetically dominated jet regions (Giannios et al. 2009; Nalewajko et al. 2011; Petropoulou et al. 2016) would be disfavoured (at least for general, non-flaring emission), and instead models based on shock waves or turbulence in weakly magnetised relativistic plasma would merit reconsideration.

Acknowledgements. We thank the referee for helpful suggestions, and Marek Sikora for comments on the manuscript. This work was supported by the Polish National Science Centre grant 2015/18/E/ST9/00580.

\section{References}

Ackermann, M., Ajello, M., Allafort, A., et al. 2011, ApJ, 743, 171 Ackermann, M., Ajello, M., Atwood, W. B., et al. 2015, ApJ, 810, 14 Błażejowski, M., Sikora, M., Moderski, R., \& Madejski, G. M. 2000, ApJ, 545, 107 
Böttcher, M., Reimer, A., Sweeney, K., \& Prakash, A. 2013, ApJ, 768, 54 Calderone, G., Sbarrato, T., \& Ghisellini, G. 2012, MNRAS, 425, L41

D’Abrusco, R., Massaro, F., Ajello, M., et al. 2012, ApJ, 748, 68

Dermer, C. D. 1995, ApJ, 446, L63

Dermer, C. D., Schlickeiser, R., \& Mastichiadis, A. 1992, A\&A, 256, L27

Dermer, C. D., Cerruti, M., Lott, B., Boisson, C., \& Zech, A. 2014, ApJ, 782, 82

Finke, J. D. 2013, ApJ, 763, 134

Fossati, G., Maraschi, L., Celotti, A., Comastri, A., \& Ghisellini, G. 1998 MNRAS, 299, 433

Ghisellini, G., \& Tavecchio, F. 2015, MNRAS, 448, 1060

Ghisellini, G., Tavecchio, F., Maraschi, L., Celotti, A., \& Sbarrato, T. 2014 Nature, 515, 376

Ghisellini, G., Righi, C., Costamante, L., \& Tavecchio, F. 2017, MNRAS, 469, 255

Giannios, D., Uzdensky, D. A., \& Begelman, M. C. 2009, MNRAS, 395, L29

Giommi, P., Padovani, P., Polenta, G., et al. 2012, MNRAS, 420, 2899

Hayashida, M., Madejski, G. M., Nalewajko, K., et al. 2012, ApJ, 754, 114

Hovatta, T., Valtaoja, E., Tornikoski, M., \& Lähteenmäki, A. 2009, A\&A, 494, 527

Janiak, M., Sikora, M., \& Moderski, R. 2015, MNRAS, 449, 431

Jester, S. 2008, MNRAS, 389, 1507

Lister, M. L., Aller, M. F., Aller, H. D., et al. 2015, ApJ, 810, L9

Madejski, G. M., \& Sikora, M. 2016, ARA\&A, 54, 725
Massaro, F., D’Abrusco, R., Ajello, M., Grindlay, J. E., \& Smith, H. A. 2011, ApJ, 740, L48

Massaro, F., Marchesini, E. J., D’Abrusco, R., et al. 2017, ApJ, 834, 113

Meyer, E. T., Fossati, G., Georganopoulos, M., \& Lister, M. L. 2011, ApJ, 740, 98

Moderski, R., Sikora, M., \& Błażejowski, M. 2003, A\&A, 406, 855

Nalewajko, K., Giannios, D., Begelman, M. C., Uzdensky, D. A., \& Sikora, M. 2011, MNRAS, 413, 333

Nalewajko, K., Begelman, M. C., \& Sikora, M. 2014a, ApJ, 789, 161

Nalewajko, K., Sikora, M., \& Begelman, M. C. 2014b, ApJ, 796, L5

Padovani, P., Giommi, P., \& Rau, A. 2012, MNRAS, 422, L48

Petropoulou, M., Giannios, D., \& Sironi, L. 2016, MNRAS, 462, 3325

Potter, W. J. 2017, MNRAS, 465, 337

Sikora, M., Begelman, M. C., \& Rees, M. J. 1994, ApJ, 421, 153

Sikora, M., Madejski, G., Moderski, R., \& Poutanen, J. 1997, ApJ, 484, 108

Sikora, M., Moderski, R., \& Madejski, G. M. 2008, ApJ, 675, 71

Sikora, M., Stawarz, Ł., Moderski, R., Nalewajko, K., \& Madejski, G. M. 2009, ApJ, 704, 38

Sironi, L., Petropoulou, M., \& Giannios, D. 2015, MNRAS, 450, 183

Tavecchio, F., \& Ghisellini, G. 2016, MNRAS, 456, 2374

Tavecchio, F., Ghisellini, G., Bonnoli, G., \& Foschini, L. 2011, MNRAS, 414, 3566

Wright, E. L., Eisenhardt, P. R. M., Mainzer, A. K., et al. 2010, AJ, 140, 1868 